\title{
ACIDENTES OCUPACIONAIS COM MATERIAL POTENCIALMENTE CONTAMINADO ENVOLVENDO TRABALHADORES DE ENFERMAGEM*
}

\author{
Gabriela da Cunha Januário ${ }^{1}$, Priscila do Carmo Freitas de Carvalho ${ }^{2}$, Graziele de Carvalho Lemos ${ }^{1}$, Elucir \\ Gir $^{3}$, Silmara Elaine Malaguti Toffano ${ }^{4}$
}

\begin{abstract}
RESUMO: Objetivou-se descrever os acidentes ocupacionais envolvendo material biológico com trabalhadores da equipe de enfermagem. Estudo descritivo, retrospectivo, com abordagem quantitativa, realizado em um hospital do Centro-Oeste Mineiro. Os dados foram coletados por meio de consulta às fichas de comunicado de acidente de trabalho e prontuários, resguardando todos os aspectos éticos. No período de outubro de 2014 a maio de 2016, 61 trabalhadores sofreram 71 exposições; 56 (91,8\%) são mulheres, 32 (52,5\%) técnicos de enfermagem, $31(50,8 \%)$ que atuavam nas enfermarias. Houve predomínio de exposições percutâneas 37 (60,7\%), em punção venosa 17 (27,9\%). Conclui-se que tais exposições poderiam ser evitadas, o que evidencia a necessidade de educação continuada para esses profissionais e a implantação de dispositivos com engenharia de segurança.

DESCRITORES: Enfermagem; Exposição ocupacional; Exposição a agentes biológicos; Riscos ocupacionais; Saúde do trabalhador.

\section{OCCUPATIONAL ACCIDENTS WITH POTENTIALLY CONTAMINATED MATERIAL INVOLVING NURSING WORKERS}

ABSTRACT: The objective was to describe the occupational accidents involving biological material involving nursing team workers. Descriptive and retrospective study with a quantitative approach, undertaken at a hospital in the Central-West of the state of Minas Gerais. The data were collected by consulting the occupational accident reporting forms and histories, safeguarding all ethical aspects. Between October 2014 and May 2016, 61 workers were victims of 71 cases of exposure; 56 (91.8\%) are women, 32 (52.5\%) nursing technicians, 31 (50.8\%) working in clinical nursing services. Percutaneous exposure $37(60.7 \%)$ during venipuncture $17(27.9 \%)$ was predominant. In conclusion, these cases of exposure could be avoided, which evidences the need for continuing education for these professionals and the implementation of devices with safety engineering.

DESCRIPTORS: Nursing; Occupational Exposure; Exposure to biological agents: Occupational risks; Occupational health.

\section{ACCIDENTES OCUPACIONALES CON MATERIAL POTENCIALMENTE CONTAMINADO INVOLUCRANDO TRABAJADORES DE ENFERMERÍA}

RESUMEN: La finalidad fue describir los accidentes ocupacionales con material biológico involucrando trabajadores del equipo de enfermería. Estudio descriptivo, retrospectivo con aproximación cuantitativa, desarrollado en un hospital del Centro-Oeste del estado de Minas Gerais. Los datos fueron recolectados mediante consulta de las fichas de informe de accidente ocupacional y archivos, salvaguardando todos los aspectos éticos. En el período de octubre del 2014 a mayo del 2016, 61 trabajadores fueron víctimas de 71 exposiciones; $56(91,8 \%)$ son mujeres, 32 (52,5\%) técnicos de enfermería, 31 (50,8\%) actuaban en las enfermarías. Predominaron exposiciones percutáneas $37(60,7 \%)$, en punción venosa $17(27,9 \%)$. Se concluye que tales exposiciones podrían ser evitadas, lo que evidencia la necesidad de educación continuada para esos profesionales y la implantación de dispositivos con ingeniería de seguridad.

DESCRIPTORES: Enfermería; Exposición ocupacional; Exposición a agentes biológicos; Riesgos ocupacionales; Salud del trabajador.

\footnotetext{
*Artigo extraído da dissertação intitulada: “Transtorno de Estresse Pós-Traumático em trabalhadores da área da saúde expostos a material biológico potencialmente contaminado". Universidade Federal de São João Del Rei, 2016.

${ }^{1}$ Enfermeira. Mestranda em Enfermagem. Universidade Federal de São João Del Rei. Divinópolis, MG, Brasil.

${ }^{2}$ Enfermeira. Mestre em Ciências. Enfermeira do Serviço de Controle de Infecção Hospitalar do Hospital Eduardo de Menezes. Belo Horizonte, MG, Brasil.

${ }^{3}$ Enfermeira. Doutora em Enfermagem. Docente da Escola de Enfermagem de Ribeirão Preto da Universidade de São Paulo. Ribeirão Preto, SP, Brasil.

${ }^{4}$ Enfermeira. Doutora em Ciências da Saúde. Docente da Universidade Federal do Triangulo Mineiro. Uberaba, MG, Brasil.
}

Autor Correspondente:

Gabriela da Cunha Januário

Universidade Federal de São João Del Rei

R. Campina Verde, 478 - 35.501-236 - Divinópolis, MG, Brasil

E-mail: gabrielacunhaj01@gmail.com
Recebido: $17 / 10 / 2016$

Finalizado: 20/12/2016 


\section{INTRODUÇÃO}

Os trabalhadores da área da saúde (TAS) estão expostos a diversos riscos ocupacionais, entre eles, destaca-se o biológico devido à constante realização de procedimentos contendo fluidos corporais ${ }^{(1-2)}$.

As exposições ocupacionais, envolvendo material biológico potencialmente contaminado (MBPC), são caracterizadas por acidentes compreendem via percutânea (ferimentos ocorridos com instrumentos perfurocortantes), mucocutânea (exposição de mucosas ocular, nasal, oral e genital e pele não íntegra) e mordeduras humanas ${ }^{(3)}$.

Segundo estudos, a principal via de exposição envolvida nos acidentes ocupacionais é a percutânea ${ }^{(4-6)}$, ocorrendo principalmente devido ao descarte inadequado de materiais perfu rocortantes, procedimentos de punção venosa e reencape de agulhas ${ }^{(2,7)}$, sendo o sangue o principal fluido envolvido ${ }^{(8-11)}$.

A equipe de enfermagem, em especial, está mais suscetível à ocorrência dessas exposições devido ao grande número de trabalhadores nesta categoria profissional, ao constante manuseio de objetos perfurocortantes e à intensa realização de cuidados diretos a clientes com diversas patologias ${ }^{(12-13)}$.

Os TAS devem estar atentos à execução de suas tarefas, sendo fundamental a adesão às PrecauçõesPadrão (PP), higiene das mãos, dentre outras medidas profiláticas, independente do diagnóstico do paciente que está sendo assistido ${ }^{(14)}$.

Embora se tenha observado grande avanço na produção científica abordando a questão da exposição ocupacional com MBPC em TAS, principalmente os de enfermagem ${ }^{(7,12)}$, poucas pesquisas foram realizadas no interior do estado de Minas Gerais.

Portanto, o presente estudo tem como objetivo descrever os acidentes ocupacionais envolvendo MBPC quanto aos aspectos profissionais, demográficos, individuais e relacionados à exposição em trabalhadores da equipe de enfermagem de um hospital filantrópico do Centro-Oeste Mineiro.

\section{METODOLOGIA}

Este estudo descritivo, retrospectivo, com abordagem quantitativa realizado em um hospital filantrópico de médio porte do Centro-Oeste Mineiro, foi aprovado pelo Comitê de Ética em Pesquisa da instituição proponente (Protocolo $n^{\circ}$ 1.349.749/2015) e da instituição Co-Participante (Protocolo ${ }^{\circ}$ 1.392.976/2015). O anonimato dos trabalhadores foi resguardado, assim como foram consideradas as diretrizes e normas regulamentadoras de pesquisas envolvendo seres humanos.

A coleta de dados consistiu na consulta às fichas de Comunicado de Acidente de Trabalho (CAT) e aos prontuários do Serviço de Engenharia, Segurança e Medicina do Trabalho (SESMT) de trabalhadores da equipe de enfermagem que se acidentaram com material biológico no período de outubro de 2014 a maio de 2016. Posteriormente, foi realizada a busca ativa destes trabalhadores de modo a convidá-los para participar desta investigação, respondendo a um instrumento estruturado, elaborado por uma das pesquisadoras, que continha questões fechadas sobre a exposição ocupacional a MBPC.

Foram incluídos na pesquisa todos os trabalhadores da equipe de enfermagem que sofreram acidente envolvendo MBPC neste período, e excluídos aqueles que na data da coleta haviam sido demitidos ou estavam em licença. Como critérios de exclusão foram adotados: trabalhadores que haviam sido demitidos ou estavam de licença no período da coleta de dados.

Os dados registrados foram codificados e digitados em planilhas do programa Excel for Windows, e posteriormente foram enviadas para o software Social Package for Social Science IBM (SPSS) ${ }^{\circledR}$, versão 22.0, e analisados por meio da estatística descritiva.

\section{RESULTADOS}


No total, $73(100 \%)$ trabalhadores da equipe de enfermagem se acidentaram com material biológico entre outubro de 2014 e maio de 2016, destes, foram excluídos sete por motivo de demissão e cinco por licença, sendo analisados, portanto ao final $61(83,5 \%)$ trabalhadores que sofreram $71(100 \%)$ exposições envolvendo MBPC neste período.

Das 71 exposições ocorridas, foi possível observar que a distribuição apresentou-se heterogênea, sendo os meses de janeiro e março de 2016 os que apresentaram maior frequência (Figura 1).

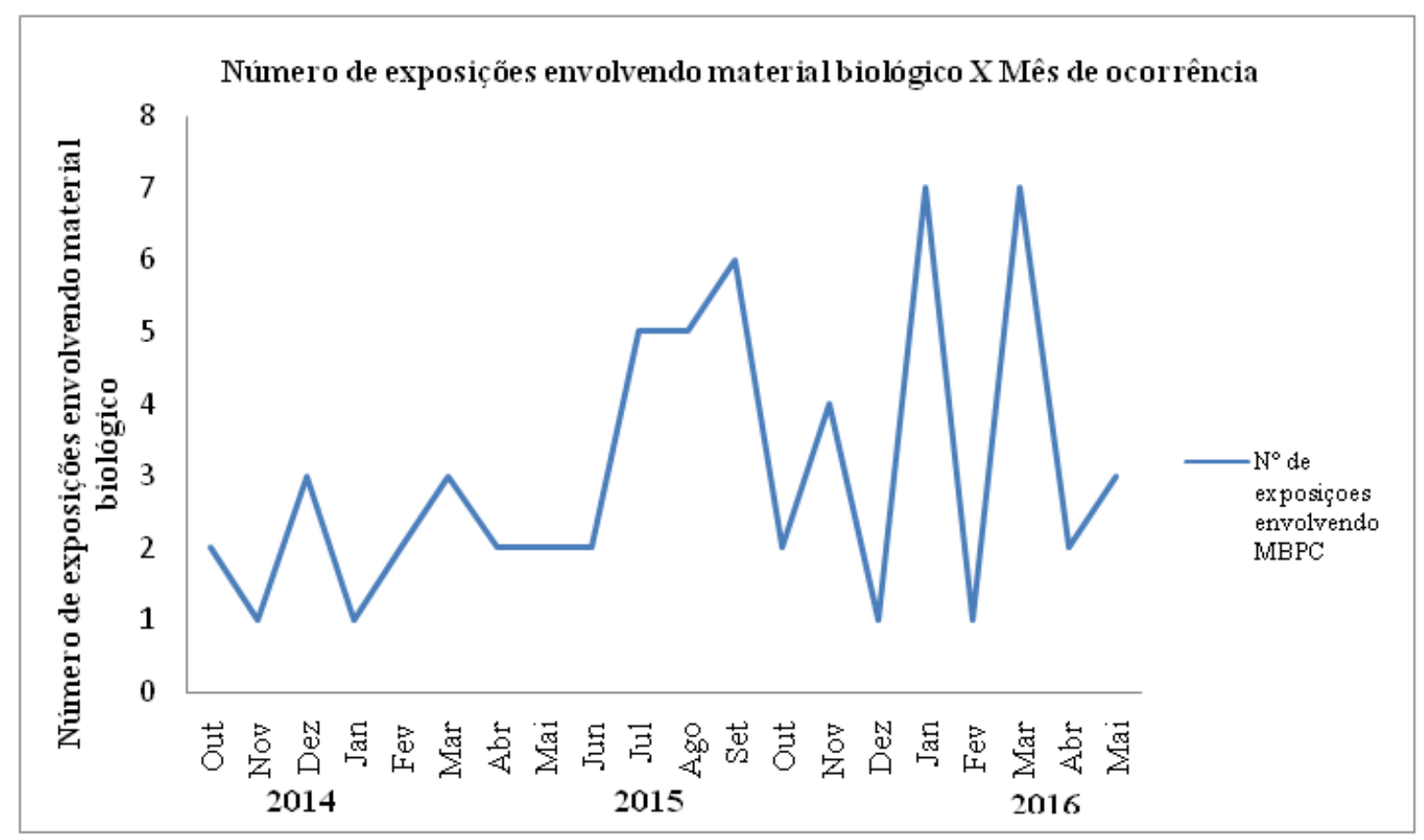

Figura 1 - Distribuição dos acidentes envolvendo material biológico potencialmente contaminado entre o período de outubro de 2014 a maio de 2016. Minas Gerais, 2016

Ao analisar as características demográficas, individuais e profissionais dos trabalhadores que participaram do estudo, as exposições ocupacionais prevaleceram entre técnicos de enfermagem $(n=32 ; 52,5 \%)$, do sexo feminino $(n=56 ; 91,8 \%)$, com média de idade de 31,4 anos e desvio-padrão de 7,5 .

Quanto ao setor de ocupação, a maioria dos acidentes ( $n=31 ; 50,8 \%)$ ocorreu na enfermaria/clínica cirúrgica; outros na Unidade de Terapia Intensiva (UTI) (n=10; 16,4\%) e no Bloco Cirúrgico (nove; 14,8\%).

Identificou-se que mais da metade dos trabalhadores contava com menos de cinco anos de experiência de trabalho na função e na instituição, $(n=40 ; 65,6 \%)$ e $(n=40 ; 65,6 \%)$, respectivamente. A maioria $(n=49 ; 80,3 \%)$ afirmou trabalhar apenas em um local.

Quanto ao número de exposições a MBPC, grande parte $(n=49 ; 80,3 \%)$ relatou ter sofrido nenhuma ou uma exposição com MBPC no último ano; assim como responderam ter recebido treinamento sobre prevenção de exposição ocupacional $(n=39 ; 63,9 \%)$. Todos $(n=61 ; 100 \%)$ estavam com esquema completo da vacina para hepatite B (Tabela 1).

Tabela 1- Características demográficas, individuais e profissionais dos TAS que sofreram acidente com material biológico no período de outubro de 2014 a maio de 2016. Divinópolis, MG, Brasil, 2016 (continua)

\begin{tabular}{llcc} 
Variável & & $\mathbf{n}$ & \% \\
\hline Sexo & Feminino & 56 & 91,8 \\
\cline { 2 - 5 } & Masculino & 5 & 8,2 \\
\hline
\end{tabular}




\begin{tabular}{|c|c|c|c|}
\hline \multirow[t]{4}{*}{ Idade } & $20-30$ & 31 & 50,8 \\
\hline & $30+40$ & 21 & 34,4 \\
\hline & $40-50$ & 6 & 9,8 \\
\hline & $\geq 50$ & 3 & 4,9 \\
\hline \multirow[t]{3}{*}{ Categoria Profissional } & Enfermeiro & 22 & 36,4 \\
\hline & Auxiliar de enfermagem & 7 & 11,5 \\
\hline & Técnico de enfermagem & 32 & 52,5 \\
\hline \multirow[t]{7}{*}{ Setor de trabalho } & Enfermaria/clínica cirúrgica & 31 & 50,8 \\
\hline & CTI Adulto/ Infantil & 10 & 16,4 \\
\hline & Bloco Cirúrgico & 9 & 14,8 \\
\hline & Maternidade/ Berçário & 4 & 6,6 \\
\hline & Oncologia & 2 & 3,3 \\
\hline & Nefrologia & 1 & 1,6 \\
\hline & Pronto- Atendimento & 4 & 6,6 \\
\hline \multirow{5}{*}{$\begin{array}{l}\text { Tempo de serviço na função } \\
\text { (em anos) }\end{array}$} & 0 -5 & 40 & 65,6 \\
\hline & 5 -10 & 13 & 21,3 \\
\hline & 10 난 & 3 & 4,9 \\
\hline & $15 \mid-20$ & 4 & 6,6 \\
\hline & $20 \mid-25$ & 1 & 1,6 \\
\hline \multirow{5}{*}{$\begin{array}{l}\text { Tempo de serviço } \\
\text { (em anos na instituição) }\end{array}$} & $0+5$ & 40 & 65,6 \\
\hline & 5 -10 & 12 & 19,7 \\
\hline & 10 난 & 5 & 8,2 \\
\hline & $15+20$ & 2 & 3,3 \\
\hline & $20-25$ & 2 & 3,3 \\
\hline \multirow[t]{2}{*}{ Vínculo em outra instituição } & Sim & 12 & 19,7 \\
\hline & Não & 49 & 80,3 \\
\hline \multirow{2}{*}{$\begin{array}{l}N^{\circ} \text { de exposições no } \\
\text { último ano de trabalho }\end{array}$} & $0-1$ & 51 & 83,6 \\
\hline & $\geq 2$ & 10 & 16,4 \\
\hline \multirow[t]{2}{*}{ Treinamento após exposição ocupacional } & Sim & 39 & 63,9 \\
\hline & Não & 22 & 36,1 \\
\hline \multirow[t]{2}{*}{ Vacinação contra hepatite B } & Sim & 61 & 100 \\
\hline & Não & 0 & 0 \\
\hline
\end{tabular}

Com relação às características referentes ao acidente ocupacional (Tabela 2), houve predomínio das exposições percutâneas $(n=37 ; 60,7 \%)$. A punção venosa $(n=17 ; 27,9 \%)$ foi citada como o procedimento de maior ocorrência, cateter intravenoso $(n=18 ; 29,5 \%)$ foi o objeto causador na maioria dos acidentes assim como o sangue $(n=43 ; 70,5 \%)$. A região mais atingida foram os dedos $(n=34 ; 55,7 \%)$.

Tabela 2 - Características relacionadas às exposições ocupacionais com material biológico potencialmente contaminado no período de outubro de 2014 a maio de 2016. Divinópolis, MG, Brasil, 2016 (continua)

\begin{tabular}{llcc} 
Variável & & $\mathbf{n}$ & $\mathbf{\%}$ \\
\hline Tipo de exposição & Mucocutânea & 24 & 39,3 \\
\cline { 2 - 4 } & Percutânea & 37 & 60,7 \\
\hline Atividade realizada & Desobstrução de acesso venoso & 3 & 4,9 \\
\cline { 2 - 4 } & Punção Venosa & 17 & 27,9 \\
\cline { 2 - 4 } & Desprezar material perfurocortante & 9 & 14,8 \\
\cline { 2 - 4 } & Administração de medicamento IM* & 10 & 16,4 \\
\hline
\end{tabular}




\begin{tabular}{|c|c|c|c|}
\hline \multirow[t]{4}{*}{ Atividade realizada } & Procedimento cirúrgico & 4 & 6,6 \\
\hline & Descarte & 1 & 1,6 \\
\hline & Reencape de agulha & 2 & 3,3 \\
\hline & Outros & 15 & 24,6 \\
\hline \multirow[t]{6}{*}{ Objeto envolvido } & Cateter intravenoso & 18 & 29,5 \\
\hline & Bisturi & 4 & 6,5 \\
\hline & Agulha & 11 & 18 \\
\hline & Escalpe & 1 & 1,6 \\
\hline & Perfurocortante não identificado & 2 & 3,2 \\
\hline & Nenhum & 25 & 40,9 \\
\hline \multirow[t]{7}{*}{ Fluido envolvido } & Secreção n/e** & 7 & 11,5 \\
\hline & Secreção n/e $\mathrm{e}^{* *}+$ Sangue & 6 & 9,8 \\
\hline & Sangue & 43 & 70,5 \\
\hline & Vômito & 1 & 1,6 \\
\hline & Urina & 1 & 1,6 \\
\hline & Líquido amniótico & 1 & 1,6 \\
\hline & Quimioterápico EV*** & 1 & 1,6 \\
\hline \multirow[t]{8}{*}{ Região atingida } & Olho & 13 & 21,3 \\
\hline & Face & 3 & 4,9 \\
\hline & Boca & 3 & 4,9 \\
\hline & Dedo & 34 & 55,7 \\
\hline & Membro Superior & 4 & 6,6 \\
\hline & Boca+ olhos+ face & 1 & 1,6 \\
\hline & Olho+ face & 2 & 3,3 \\
\hline & Face+ mucosa nasal & 1 & 1,6 \\
\hline
\end{tabular}

* Intramuscular

** não especificada

***endovenoso

Quanto às características envolvendo situação sorológica do paciente-fonte e condutas pósexposição ocupacional, a maioria dos clientes apresentou sorologia negativa para HIV e VHC ( $n=47$; $77,0 \%)$, mais da metade dos trabalhadores envolvidos não recebeu antirretroviral $(n=53 ; 86,9 \%)$, todos relataram o acidente ao SESMT $(n=61 ; 100 \%)$ e a maioria $(n=48 ; 78,7 \%)$ não foi avaliada por serviço de infectologia especializado (Tabela 3).

Tabela 3 - Características das exposições ocupacionais com material biológico quanto à sorologia do pacientefonte no momento do acidente, procura por atendimento médico especializado e uso de antirretrovirais. Divinópolis, MG, Brasil, 2016 (continua)

\begin{tabular}{|c|c|c|c|}
\hline Variável & & n & $\%$ \\
\hline \multirow{7}{*}{$\begin{array}{l}\text { Situação sorológica } \\
\text { do paciente- fonte }\end{array}$} & HIV - VHC - & 47 & 77 \\
\hline & $\mathrm{HIV}+\mathrm{VHC}+$ & 1 & 1,6 \\
\hline & $\mathrm{HIV}+\mathrm{VHC}-$ & 2 & 3,2 \\
\hline & HIV - VHC + & 4 & 6,5 \\
\hline & HIV - VHC s/i* & 1 & 1,6 \\
\hline & $\mathrm{HIV}+\mathrm{VHC} \mathrm{s} / \mathrm{i}^{*}$ & 1 & 1,6 \\
\hline & $\mathrm{s} / \mathrm{i}$ & 5 & 8,1 \\
\hline \multirow[t]{2}{*}{ Recebeu antirretroviral } & Sim & 8 & 13,1 \\
\hline & Não & 53 & 86,9 \\
\hline
\end{tabular}




\begin{tabular}{llll}
\hline $\begin{array}{l}\text { Avaliação por serviço de } \\
\text { infectologia especializado }\end{array}$ & Sim & 13 & 21,3 \\
\cline { 2 - 4 } & Não & 48 & 78,7
\end{tabular}

* sem informações

\section{DISCUSSÃO}

No presente estudo, observou-se um predomínio de exposições ocupacionais envolvendo técnicos de enfermagem e esses dados foram semelhantes aos encontrados em outro estudo ${ }^{(9)}$. Isso é justificado pelo maior volume dos trabalhadores da equipe de enfermagem nas instituições de saúde, sendo que $80 \%$ são compostos por técnicos e auxiliares e $20 \%$ enfermeiros ${ }^{(15)}$.

O sexo feminino representou a maioria dos expostos. Isto se deve às próprias características históricas da equipe de enfermagem que em sua grande maioria é exercida por mulheres ${ }^{(8)}$, justificando assim sua maior prevalência e corroborando com outras pesquisas encontradas na literatura nacional e internacional ${ }^{(4,11,13)}$.

No Brasil, estudo nacional realizado pela Fundação Oswaldo Cruz (FIOCRUZ), em iniciativa do Conselho Federal de Enfermagem (COFEN), estimou que a equipe de enfermagem seja composta por $84,6 \%$ de mulheres e que embora este número seja muito superior ao sexo masculino, está havendo uma tendência de masculinização da classe desde os anos de $1990^{(15)}$.

A faixa etária de 20 a 30 anos foi a maior em número de exposições ocorridas entre os entrevistados, o que corrobora com outros estudos ${ }^{(6,11)}$. A faixa etária jovem também foi mais evidenciada em outro achado ${ }^{(16)}$ e justificada pelo fato de que trabalhadores acima de 40 anos executam mais tarefas administrativas e menos ações relacionais a coleta de sangue ou outras situações associadas a risco mais elevado para exposições ocupacionais. Em contrapartida outro autor ${ }^{(17)}$ revela preocupação com relação a essa faixa etária que em tão pouco tempo já se expôs a MBPC.

Quanto ao setor de ocorrência desses acidentes, a enfermaria/clínica cirúrgica representou mais da metade das exposições, divergindo dos resultados encontrados na literatura que apontam o bloco cirúrgico como o setor de maior ocorrência para acidentes ocupacionais ${ }^{(18-19)}$.

Em relação ao tempo de experiência profissional na função e na instituição, a maioria dos entrevistados referiu tempo de trabalho menor que cinco anos, corroborando com outro achado ${ }^{(20)}$. Divergido desses resultados, um estudo apresentou taxas elevadas de acidentes em trabalhadores com mais de 10 anos na instituição ${ }^{(8)}$, não nos permitindo chegar a um consenso com relação à questão da experiência profissional na ocorrência de exposição envolvendo MBPC.

Quanto à situação vacinal dos acidentados, todos estavam com esquema vacinal completo para hepatite B no momento da admissão na instituição, de acordo com dados de seus prontuários, tal como evidenciado em outra pesquisa ${ }^{(21)}$. Em um estudo realizado com trabalhadores do Rio Grande do Norte, mais da metade dos profissionais expostos a MBPC estava vacinados para hepatite B, entretanto não se tinha informações a respeito da resposta vacinal, não podendo descartar, portanto o risco de menor taxa de imunoproteção quando avaliada pela obtenção de anticorpos anti-HBs ${ }^{(9)}$.

Outro achado evidenciou que entre os trabalhadores expostos a MBPC, o maior percentual de profissionais não vacinados foi de técnicos e auxiliares de enfermagem, seguidos de profissionais da higienização e limpeza, representando $4,3 \%$ e $2,2 \%$, respectivamente ${ }^{(2)}$. Isto mostra a necessidade de conscientização dos TAS com relação à vacinação, já que a hepatite B é uma doença imunoprevinível.

Quanto ao tipo de exposição, mais da metade envolveu a via percutânea. Esses dados foram semelhantes à pesquisa realizada na Polônia ${ }^{(4)}$ em que houve predominância de acidentes com perfurocortante em $92.5 \%$ dos casos e em outros achados encontrados ${ }^{(5-6,10,22)}$.

Os acidentes aconteceram em diversas situações de prestação de cuidado aos clientes, porém o procedimento de punção venosa foi o mais relatado pelos entrevistados. Resultado semelhante 
encontrado em um hospital do interior paulista, quando $29,1 \%$ das exposições ocorreram durante realização desta atividade ${ }^{(23)}$. Em uma pesquisa realizada em 50 municípios da região sul de Minas Gerais, o principal preditor para ocorrência de exposições ocupacionais foi o descarte inadequado de materiais $^{(2)}$.

O objeto mais utilizado nos acidentes foi o cateter intravenoso, o que diferiu de alguns estudos, que apresentaram a agulha oca como principal material envolvido nas exposições ocupacionais ${ }^{(22,24)}$.

Como alternativa para minimização de riscos envolvendo acidentes com materiais perfurocortantes, é necessário que sejam disponibilizados dispositivos seguros com engenharia de segurança, com o intuito de diminuir as exposições dos TAS a diversos patógenos que podem ser transmitidos ${ }^{(25-27)}$. Tais dispositivos foram recomendados e passaram a ser de uso obrigatório em todos os serviços de saúde brasileiros.

O sangue foi o fluido corporal mais envolvido, concordando com outro estudo ${ }^{(10)}$ que evidenciou o material biológico em 78,9\% dos acidentes. Outros achados na literatura também encontraram este fluido como o mais presente nas exposições ${ }^{(9,11,22)}$. Quanto à região, o local mais atingido foi o dedo seguido dos olhos, situação semelhante à encontrada ${ }^{(28)}$ em que $56,4 \%$ dos acidentes envolveram dedos da mão e $32,5 \%$ olhos.

Quanto à situação sorológica, na maioria dos acidentes o paciente-fonte foi identificado, corroborando com outro estudo ${ }^{(2)}$.

Em relação à conduta adotada após exposição, grande maioria dos entrevistados não realizou consulta em serviço de infectologia especializado, pois segundo relatos dos participantes, foi descartada a possibilidade de infecção após consulta feita no pronto-atendimento do próprio hospital; a minoria dos expostos usou antirretroviral. Estudo encontrado na literatura evidencia a não procura por atendimento médico e taxa de abandono do seguimento clínico ${ }^{(8)}$.

Em pesquisa realizada foi identificada, através de análise de regressão logística, que exposições cuja fonte era conhecida e com sorologia negativa os profissionais tiveram 29 vezes maior chance de aderir ao tratamento se comparado aqueles cujo paciente-fonte era desconhecido ou com sorologia positiva $^{(6)}$.

\section{- CONCLUSÃO}

Concluiu- se que a maioria dos acidentes ocorreu entre técnicos de enfermagem, do sexo feminino, com média de idade de 31,4 anos, nos setores de enfermaria/clínica cirúrgica. Houve predomínio das exposições percutâneas, durante realização de procedimentos de punção venosa, sendo o sangue o fluido corporal presente em mais da metade das exposições e a região do dedo a mais atingida. Grande parte dos acidentados não foi atendida em um serviço especializado e todos os trabalhadores estavam com esquema vacinal completo para hepatite B.

A partir dos resultados encontrados, pode-se observar que embora existam diversas medidas de biossegurança, ainda existe um número preocupante de exposições ocupacionais envolvendo MBPC com trabalhadores da equipe de enfermagem, que devido às próprias características do seu trabalho estão mais suscetíveis a estas ocorrências. Portanto, é necessária educação continuada destes profissionais e também a implantação pelos serviços de saúde de dispositivos seguros com engenharia de segurança, visando assim à minimização destas ocorrências.

Este estudo tem como limitações as lacunas na ficha de preenchimento do acidente com variáveis relacionadas à exposição a material biológico e ainda o fato dos dados se referirem a um hospital específico, não podendo ser generalizados.

\section{- REFERÊNCIAS}

1. Valim MD, Marziale MHP, Hayashida M, Richart-Martínez M. Occurrence of occupational acidentesinvolving potentially contaminated biological material among nurses. Acta paul.enferm.[Internet] 2014;27(3) [acesso em 04 
ago 2016]. Disponível: http://dx.doi.org/10.1590/1982-0194201400047.

2. Julio RS, Filardi MBS, Marziale MHP. Acidentes de trabalho com material biológico ocorridos em municípios de Minas Gerais. Rev. bras. enferm. [Internet] 2014;67(1) [acesso em 15 jul 2016]. Disponível: http://dx.doi. org/10.5935/0034-7167.20140016.

3. Ministério do Trabalho e Emprego, Secretaria de Inspeção do Trabalho (BR). Departamento de Segurança e Saúde no Trabalho. Guia de Análise Acidentes de Trabalho. Brasília: Ministério do Trabalho e Emprego, Secretaria de Inspeção do Trabalho; 2010.

4. Rymer W, Gladysz A, Filipowski H,Zubkiewicz-Zarebska A, Tuminska A, KnyszB. Risk of occupational exposure to the HBV infection in non-clinical healthcare personnel.Med Pr. [Internet] 2016;67(3) [acesso em 10 set 2016$].$ Disponível: http://dx.doi.org/10.13075/mp.5893.00272.

5. Khalil SS, Khalil OAK,Lopes-Júnior LC, Cabral DB, Bomfim, EO, Landucci LF, et al. Occupational exposure to bloodborne pathogens in a specialized care service in Brazil. Am. J. Infect. Control. [Internet] 2015;43(8) [acesso em 17 jun 2016]. Disponível: http://dx.doi.org/10.1016/j.ajic.2015.05.030.

6. de AlmeidaMCM, Canini SRMS, Reis RK, Malaguti-ToffanoSE, Pereira FMV, Gir E.Seguimento clínico de profissionais e estudantes da área da saúde expostos a material biológico potencialmente contaminado. Rev. esc. enferm.USP. [Internet] 2015;49(2) [acesso em 25 set 2016]. Disponível: http://dx.doi.org/10.1590/S0080623420150000200011.

7. Alves AP,Ferreira MD, Prearo MF, Gir E, Canini SRMS.Subnotificação de acidentes ocupacionais com material biológico pela enfermagem no bloco cirúrgico. Rev. Eletr. Enf. [Internet] 2013;15(2) [acesso em 18 ago 2016$].$ Disponível: http://dx.doi.org/10.5216/ree.v15i2.18554.

8. Pimenta FR, Ferreira MD, Gir E, Hayashida M, Canini SRMS. Atendimento e seguimento clínico especializado de profissionais de enfermagem acidentados com material biológico. Rev. esc. enferm. USP. [Internet] 2013;47(1) [acesso em 30 ago 2016]. Disponível: http://dx.doi.org/10.1590/S0080-62342013000100025.

9. Cavalcante CAA, Cavalcante EFO, Macêdo MLAF, Cavalcante ES, de Medeiros SM. Acidentes com material biológico com trabalhadores. Rev. Rene. [Internet]2013;14(5) [acesso 01 ago 2016]. Disponível: http://www. periodicos.ufc.br/index.php/rene/article/viewFile/3631/2873.

10. Giancotti GM, Haeffner R, Solheid NLS, Miranda FMDA, Sarquis LMM. Caracterização das vítimas e dos acidentes de trabalho com material biológico atendidas em um hospital público do Paraná, 2012. Epidemiol. Serv. Saúde. [Internet] 2014;23(2) [acesso em 02 jul 2016]. Disponível: http://dx.doi.org/10.5123/S167949742014000200015.

11. Luize PB, Canini SRMS, Gir E, Malaguti-Toffano SE. Condutas após exposição ocupacional a material biológico em um hospital especializado em oncologia. Texto Contexto Enferm. [Internet] 2015;24(1) [acesso em 14 jun 2016].Disponível: http://dx.doi.org/10.1590/0104-07072015002700013.

12. Soares LG, Sarquis LMM, Kirchhof ALC, Cruz EDA. Percepção do risco biológico em trabalhadores de enfermagem. CogitareEnferm. [Internet] 2013;18(1) [acesso em 22 jun 2016]. Disponível: http://dx.doi.org/10.5380/ ce.v18i1.31300.

13. Malaguti-Toffano SE, Canini SRMS, Reis RK, Pereira FMV, Felix MAS, Ribeiro PHV, et al. Adesão às precauçõespadrão entre profissionais da enfermagem expostos a material biológico. Rev. Eletr. Enf. [Internet]2015;17(1) [acesso em 07 ago 2016]. Disponível: http://dx.doi.org/10.5216/ree.v17i1.29269.

14. de Lima LM, de Oliveira CC, de Rodrigues KMR. Exposição ocupacional por material biológico no Hospital Santa Casa de Pelotas- 2004 a 2008. Esc. Anna Nery. [Internet] 2011;15(1) [acesso em 19 set 2016]. Disponível: http://dx.doi.org/10.1590/S1414-81452011000100014.

15. Fundação Oswaldo Cruz (FIOCRUZ). Conselho Regional de Enfermagem (COFEN). Pesquisa inédita traça perfil da enfermagem. [Internet] 2015 [acesso em 29 set 2016] Disponível: http://www.cofen.gov.br/pesquisainedita-traca-perfil-da-enfermagem_31258.html.

16. Yoshikawa T, Wada K, Lee JJ, Mitsuda T, Kidouchi K, Kurosu H, et al.Incidence rate of needlestick and sharps injuries in 67 japanese hospitals: a national surveillance study.PLos ONE. [Internet] 2013;8(10) [acesso em 11 jul 
2016]. Disponível: http://dx.doi.org/10.1371/journal.pone.0077524.

17. Nowak NL, Campos GA, Borba EO, Ulbricht L, Neves EB. Fatores de risco para acidentes com materiais perfurocortantes. Mundo saúde. [Internet] 2013;37(4) [acesso em 26 ago 2016]. Disponível: http://www.saocamilosp.br/pdf/mundo_saude/155558/A06.pdf.

18. de Souza-Borges FRF, Ribeiro LA, de Oliveira LCM.Occupational exposures to body fluids and behaviors regarding their prevention and post-exposure among medical and nursing students at a brazilian public university. Rev. Inst. Med. Trop. [Internet]2014;56(2) [acesso em 27 ago 2016]. Disponível: http://dx.doi.org/10.1590/S003646652014000200012.

19. Afridi AAK, Kumar A, Sayani R. Needle stick injuries- risk and preventive factors: a study amng health care intertiary care hospitals in Pakistan. Glob J Health Sci. [Internet] 2013;5(4) [acesso em 22 ago 2016]. Disponível: http://dx.doi.org/10.5539/gjhs.v5n4p85.

20.Al-Khatib IA, El Ansari W, Aregat TA, Darkhawaia RA, Mansour SH, Tucktuck MA, et al. Occupational safety precautions among nurses at four hospitals, Nablus district, Palestine. Int. J. Occup. Med. Environ. Health. [Internet] 2015;6(4) [acesso em 03 set 2016]. Disponível: http://www.theijoem.com/ijoem/index.php/ijoem/ article/view/581.

21.Tipple ACFV, Silma EAC, Teles AS, Mendonça KM, Silva e Souza AC, Melo DS. Acidente com material biológico no atendimento pré-hospitalar móvel: realidade para trabalhadores da saúde e não saúde. Rev. bras. enferm. [Internet] 2013;66(3) [acesso em 19 ago 2016]. Disponível: http://dx.doi.org/10.1590/S0034-71672013000300012.

22. Santos SS, da Costa NA, Mascarenhas MDM. Caracterização das exposições ocupacionais a material biológico entre trabalhadores de hospitais no Município de Teresina, Estado do Piauí, Brasil, 2007 a 2011. Epidemiol. Serv. Saúde. [Internet] 2013;22(1) [acesso em 11 ago 2016]. Disponível: http://dx.doi.org/10.5123/ S1679-49742013000100017.

23. Marziale MHP, dos Santos HEC, Cenzi CM, Rocha FLR, Trovó MEM. Consequências da exposição ocupacional a material biológico entre trabalhadores de um hospital universitário. Esc. Anna Nery. [Internet] 2014;18(1) [acesso em 13 ago 2016]. Disponível: http://dx.doi.org/10.5935/1414-8145.20140002.

24. Samargandy SA, Bukhari LM, Samargandy SA, Bahlas RS, Aldigs EK, Alawi MA, et al. Epidemiology and clinical consequences of occupational exposure to blood and other body fluids in a university hospital in Saudi Arabia. Saudi MedJ. [Internet] 2016;37(7) [acesso em 14 jul 2016]. Disponível: http://dx.doi.org/10.15537/smj.2016.7.14261.

25. Beynon A. A quality improvement initiative to reduce needlestick injuries.Nurs Stand. [Internet] 2015;29(22) [acesso em 14 jul 2016]. Disponível: http://dx.doi.org/10.7748/ns.29.22.37.e9471.

26. Ministério do Trabalho e Emprego (BR). Portaria n. 939 de novembro de 2008. Regulamenta o cronograma previsto no item 32.2.4.16 da Norma Regulamentadora n. 32 (NR 32). Diário Oficial da União, [Internet] 19 nov 2008b. Seção 1 [acesso em 01 jul 2016]. Disponível: http://www.trtsp.jus.br/geral/tribunal2/ORGAOS/MTE/ Portaria/P939_08.html.

27. Ministério do Trabalho e Emprego (BR).Portarian. 1.748. Diário Oficialda União, 30 de outubro de 2011: regulamenta o subitem 32.2.4.16 da Norma Regulamentadora n. 32.Diário Oficial da União, [Internet] 31 ago 2011 [acesso em 02 jul 2016]. Disponível: http://www.trtsp.jus.br/geral/tribunal2/ORGAOS/MTE/Portaria/P1748_11. html.

28. de Carvalho PCF, Januário GC, Lopes LP, Reis RK, Malaguti-Toffano SE. Exposição a material biológico envolvendo trabalhadores em hospital especializado em doenças infecciosas. Rev. baiana enferm. [Internet] 2016;30(3) [acesso em 26 jul 2016]. Disponível: http://dx.doi.org/10.18471/rbe.v30i3.15670. 